\title{
Literature Review on Tongue Pressure of Healthy Japanese
}

\author{
Keiji Tabuchi $^{1, ~ *}$, Kensuke Norikoshi ${ }^{1,2}$, Toshio Kobayashi ${ }^{1}$ \\ ${ }^{1}$ Institute of Bio-Medical and Health Sciences, Hiroshima University, Hiroshima, Japan \\ ${ }^{2}$ Faculty of Nursing, Hiroshima International University, Hiroshima, Japan \\ Email address: \\ tabk2@hirosima-u.ac.jp (K. Tabuchi), k-muraki@ns.hirokoku-u.ac.jp (K. Norikoshi), tkobaya@hiroshima-u.ac.jp (T. Kobayashi) \\ ${ }^{*}$ Corresponding author
}

\section{To cite this article:}

Keiji Tabuchi, Kensuke Norikoshi, Toshio Kobayashi. Literature Review on Tongue Pressure of Healthy Japanese. Science Journal of Public Health. Vol. 6, No. 3, 2018, pp. 86-90. doi: 10.11648/j.sjph.20180603.14

Received: April 18, 2018; Accepted: May 8, 2018; Published: June 21, 2018

\begin{abstract}
The tongue plays an important role in speaking, eating, and drinking while making complicated movements in the oral cavity. By measuring tongue pressure, it is possible to infer the extent of tongue function. In the previous study, there were several papers on tongue pressure which examined subjects by disease, such as symptoms of temporomandibular joint disease, aspiration, but there are few studies on measuring tongue pressure of healthy Japanese. Additionally, there is no study on all age focusing on the relationship between tongue pressure and age and gender. Therefore, the purpose of this study was to reveal the tendency of healthy Japanese tongue pressure through literature review. Japanese medicine abstract society web service and PubMed were used to search the keyword "tongue pressure" in English and Japanese, 58 English papers and 291 Japanese papers were extracted. There were 34 papers including Japanese tongue pressure data. The text and abstract were carefully read and the targets were 12 papers including healthy Japanese tongue pressure data. Data sorted tongue pressure by gender and age. Boys and girls mixed tongue pressure from neonates to three month old was 10-13 $\mathrm{kPa}, 29-35 \mathrm{kPa}$ for elementary school students, $35 \mathrm{kPa}$ for junior high school students and high school students. Up to high school students, there was no document that measured tongue pressure by gender, respectively. Since the difference between men and women growth begins with middle of elementary school age, it is considered that necessary to measure tongue pressure according to gender. The tongue pressure of women in their twenties to sixtieth was 33-40 kPa, which was the peak of Japanese women's tongue pressure. Twenties men's tongue pressure is 45 to $55 \mathrm{kPa}$, which was the peak of Japanese men's tongue pressure. The maximum tongue pressure in lifetime was found to be 5 to $22 \mathrm{kPa}$ higher for men than for women. However, in women, peak tongue pressure was kept until sixtieth, and the subsequent decrease in tongue pressure was moderate compared to men. In the case of men, the decline in tongue pressure starts in the forties, almost the same tongue pressure as women in the seventies, tongue pressure in men in their octogenarian was inferior to that of the same age women. In literature review, gender and age characteristics and research problems on healthy Japanese tongue pressure were clarified.
\end{abstract}

Keywords: Literature Review, Tongue Pressure, Healthy Japanese

\section{Introduction}

The tongue plays an important role in speaking, eating, and drinking while making the complicated movements in the oral cavity, deterioration of the tongue function means a decrease in nutrition intake and communication ability [1-3]. Therefore, strengthening and maintaining the movement function of the tongue is important for improving the quality of life. Recently, attention has been focused on measuring tongue pressure as an index for objectively evaluating the motor function of the tongue [4]. Tongue pressure measurement can be conveniently carried out by pushing a balloon-like probe between the palate and the tongue. Tongue Pressure's measuring equipment has been commercialized in Japan since 2012. In the previous study, there were several papers on tongue pressure which examined subjects by disease, such as symptoms of temporomandibular joint disease [5-6], but there have been few studies for healthy Japanese. Additionally, there is no study on all age focusing on the relationship between tongue pressure and age and gender. Oral function declines with age. It is important to know the transition of the motor function of the tongue of a 
healthy growing person in order to support a person whose tongue movement has declined.

\section{Aim}

Aim of this retrospective study is to clarify the features of tongue pressure of healthy Japanese and research problems from literature review.

\section{Method}

Extensive review of the recent literature was conducted in electronic database PubMed and Japan Medical Abstracts Society Web Service, a search was conducted with the keyword "tongue pressure" in Japanese, and the results of 291 papers were obtained. PubMed searched with the keywords "tongue pressure" and "Japanese" in English, and the results of 58 papers were obtained. References from original paper to conference proceedings were targeted. Inclusion criteria is a paper that includes data on healthy Japanese's tongue pressure and even if the essence of the paper is for the study of a specific disease, when the tongue pressure data of the healthy group to be compared exists, only data of healthy group was adopted as subject data of this study. Exclusion criteria were data on patients with specific diseases such as bone abnormality around the oral cavity, aspiration, etc. Only the healthy Japanese tongue pressure values sorted by gender and age were carefully read and extracted from the papers and abstract.

\section{Result}

\subsection{Result of Literature Research}

58 English papers and 291 Japanese papers were searched. There were 34 papers including Japanese tongue pressure data. The paper and abstract were carefully read and the target was 12 papers including healthy Japanese tongue pressure data. The authors of 12 papers, the age of healthy Japanese subjects, the number of participants, the gender, values of tongue pressure, etc. are summarized in Table 1. Data sorted tongue pressure by gender and age.

Table 1. List of target literature.

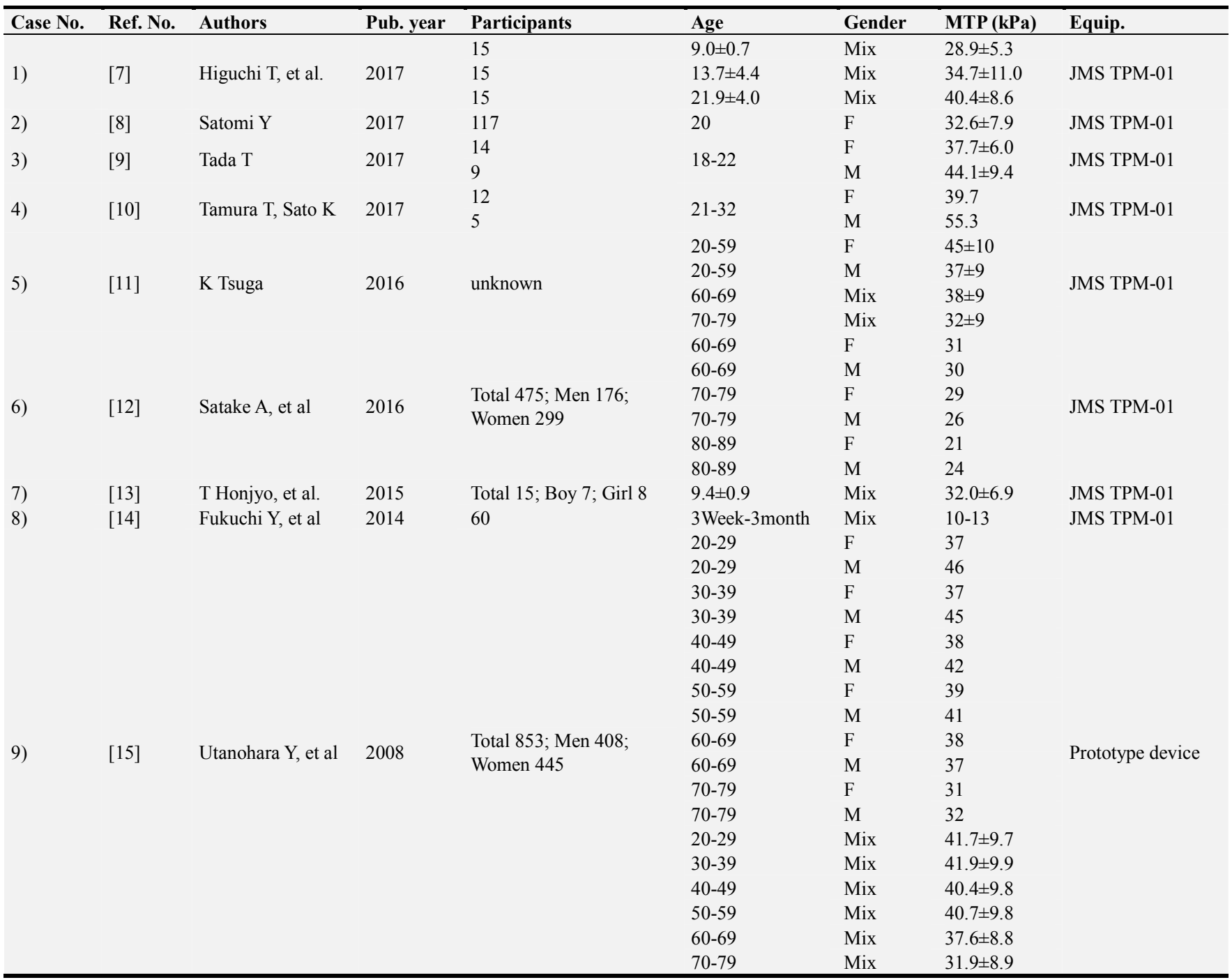




\begin{tabular}{|c|c|c|c|c|c|c|c|c|}
\hline Case No. & Ref. No. & Authors & Pub. year & Participants & Age & Gender & MTP (kPa) & Equip. \\
\hline \multirow{2}{*}{ 10) } & \multirow{2}{*}{ [16] } & \multirow{2}{*}{ Tsuga $\mathrm{K}$, et al. } & \multirow{2}{*}{2011} & 20 & \multirow{2}{*}{$21-32$} & F & 34 & \multirow{2}{*}{ ALMIC TPS-300 } \\
\hline & & & & 20 & & M & 48 & \\
\hline 11) & {$[17]$} & Yoshida M, et al. & 2006 & $\begin{array}{l}109 \\
36\end{array}$ & $64-100$ & $\begin{array}{l}\mathrm{F} \\
\mathrm{M}\end{array}$ & $\begin{array}{l}15.9 \pm 1.0 \\
15.6 \pm 1.7\end{array}$ & Prototype device \\
\hline 12) & {$[18]$} & M Kodama, et al. & 2004 & 83 & $82.0 \pm 7.7$ & Mix & $22.1 \pm 9.3$ & Prototype device \\
\hline
\end{tabular}

M, Men; F, women; Mix, Including men and women data; MTP, Max tongue pressure

\subsection{Organization of Tongue Pressure Values by Gender and Age}

\subsubsection{Men Age-Specific Tongue Pressure Value}

Based on the contents of 12 papers, as a result of organizing the tongue pressure value of only men by age, "Range of tongue pressure value of healthy Japanese Men" is shown in the Figure 1. Seven papers described men's tongue pressure. The vertical axis is tongue pressure value $(\mathrm{kPa})$, and the horizontal axis is age range. The color difference in the figure shows Case number.

The tongue pressure of a healthy Japanese men was as follows. There was no paper showing only men's data from neonates to middle of teenage. From end of teenage to beginning of twenties was $45-55 \mathrm{kPa}$. In thirties it was $45 \mathrm{kPa}$. From forties to fifties it was $41-45 \mathrm{kPa}$. It decreased to 31 $37 \mathrm{kPa}$ for sixties and further decreased to $29-33 \mathrm{kPa}$ for seventies. It decreased to $15-21 \mathrm{kPa}$ in octogenarian and 15 $\mathrm{kPa}$ in nonagenarian.

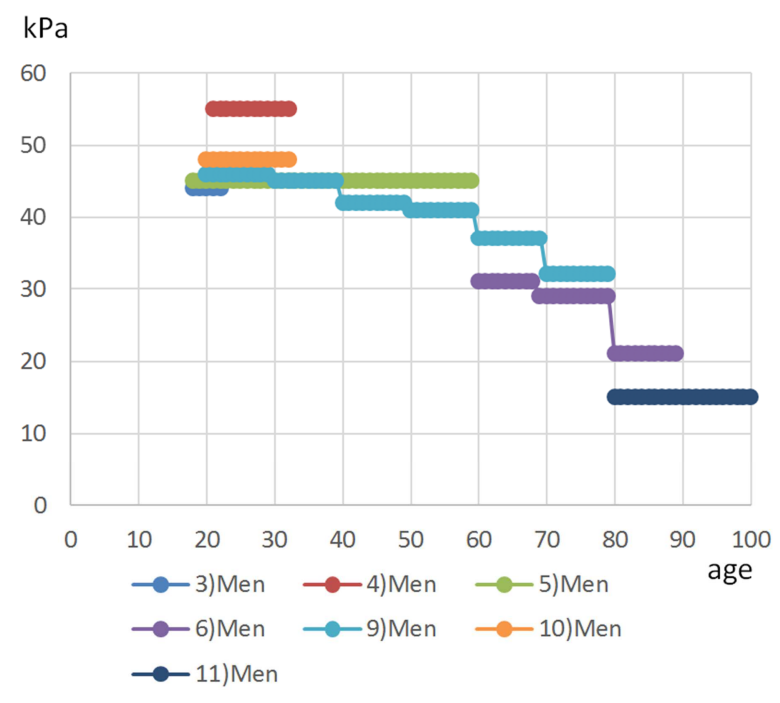

Figure 1. Range of tongue pressure value of healthy Japanese Men.

\subsubsection{Women Age-Specific Tongue Pressure Value}

Based on the contents of 12 papers, as a result of organizing the tongue pressure value of only women by age, "Range of tongue pressure value of healthy Japanese Women" is shown in the Figure 2. Eight papers described Women's tongue pressure. The tongue pressure of a healthy Japanese women was as follows. There was no paper showing only women's data from neonates to middle of teenage. Tongue pressure from end of teenage to beginning of thirties was $33-40 \mathrm{kPa}$. From thirties to fifties it was 37-40 $\mathrm{kPa}$. In the case of Japanese women, the decrease in tongue pressure started in the sixties and it is 30 to $38 \mathrm{kPa}$. The drop in tongue pressure was $26-31 \mathrm{kPa}$ in the seventies, $16-24$ $\mathrm{kPa}$ in octogenarian and $16 \mathrm{kPa}$ in nonagenarian.

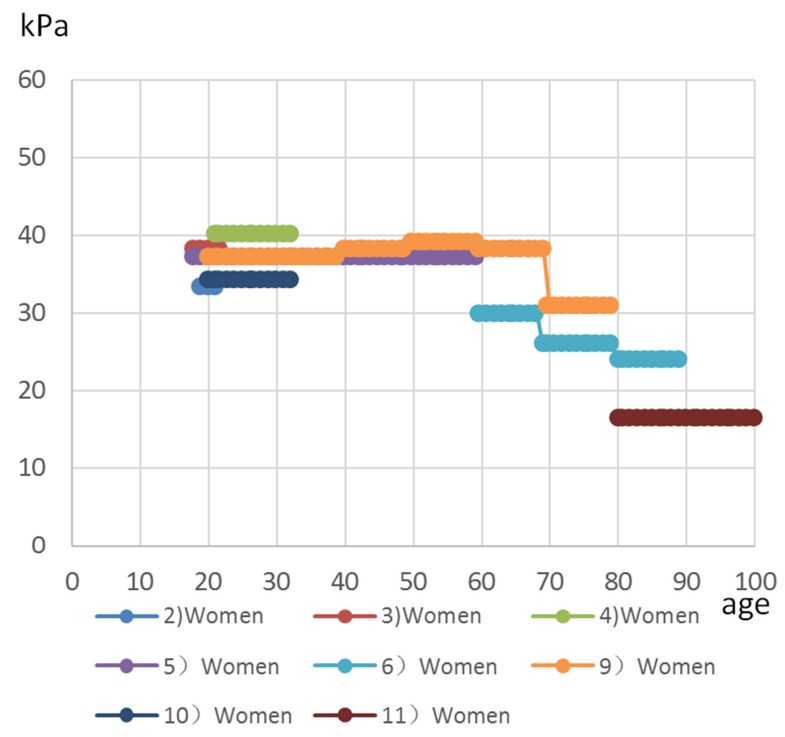

Figure 2. Range of tongue pressure value of healthy Japanese Women.

\subsubsection{Tongue Pressure Values by Gender and Age (Mixed Data of Men and Women)}

Based on the contents of 12 papers, as a result of organizing the tongue pressure value of men and women mixed data by age, "Range of tongue pressure value of healthy Japanese mix data (men and women)" is shown in the Figure 3. In the six papers, mixed data of men and women tongue pressure were shown. Since it cannot be divided by gender as shown in Figure 1 and Figure 2, it is shown as mix data.

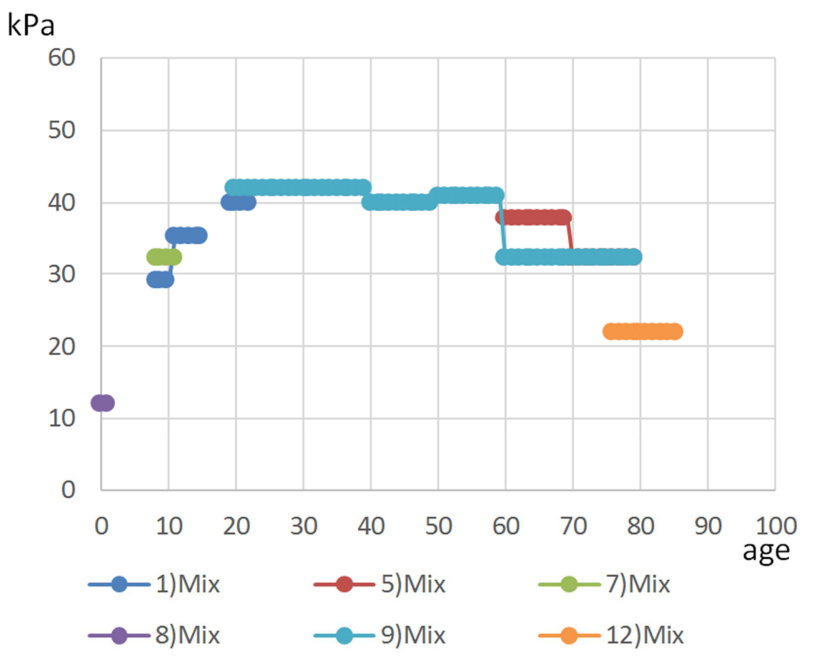

Figure 3. Range of tongue pressure value of healthy Japanese Mix Data (Women \& Men). 
Men and women mixed tongue pressure of neonates from 3 weeks to 3 months after birth was $10-13 \mathrm{kPa}$. There was no paper showing mix data from 3 months old to lower class of primary school. In the middle class of primary school, the mixed tongue pressure for boys and girls were $29-32 \mathrm{kPa}$, and in the upper class of primary school it was $32-35 \mathrm{kPa}$. Men and women mixed tongue pressure of junior high school students and high school students was $35 \mathrm{kPa}$.

\section{Discussion}

Tongue pressure of infants aged 1 to 3 months were 10 to $13 \mathrm{kPa}$. Because infants can drink milk, tongue pressure around $10 \mathrm{kPa}$ was considered necessary to ingest liquid from the mouth. If the tongue pressure value was more than $10 \mathrm{kPa}$, the lips can be closed, the function of the epiglottis is maintained, if the person is willing to take nutrition and moisture from the mouth, not nasal ductal nutrition or intravenous nutrition, It was considered possible to consider the supplementation of moisture by oral ingestion or the use of drink-like nutrient.

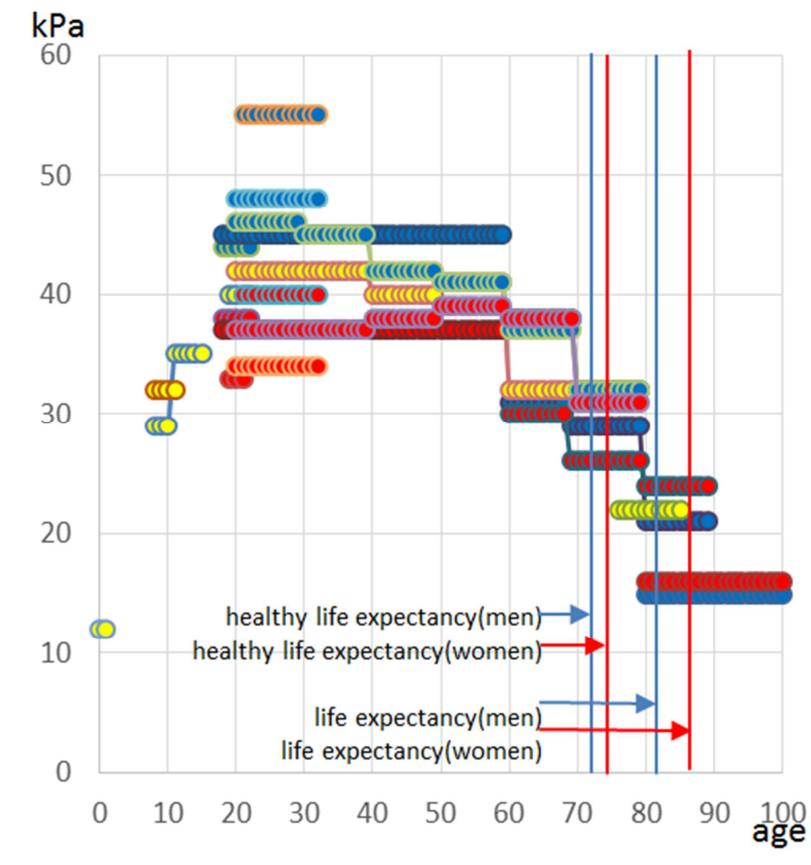

Figure 4. Range of tongue pressure value of healthy Japanese All Data.

There was no tongue pressure data of healthy Japanese children between 1 and 8 years old. Transition from milk to baby food and solid foods has started in the data blank period. It is time for daily conversation by speech to become possible at this period. Tongue pressure data of this period is necessary. Consideration of the shape of food and the necessary tongue's power, to consider the relationship between tongue movement and tongue pressure by speech.

The tongue pressure values of high school students and below were only data measured together with men and women. And papers were not many. Generally, Growth difference between boys and girls at the early stage of elementary school is small. However, in the upper class of primary school, girls grow faster than boys. In the junior high school, the growth of boy is accelerated and it is common to exceed the physique of girl [19]. It is necessary to acquire data of this period in order to clarify the relation between the growth of the body and tongue pressure. Therefore, it is considered that it is necessary to measure tongue pressure separately for boy and girl from elementary school grade.

Women from end of teenage to sixties kept tongue pressure of $33-40 \mathrm{kPa}$, this age was the peak of the tongue pressure value of Japanese women. On the other hand, men from end of teenage to twenties maintained tongue pressure of 45-55 $\mathrm{kPa}$, this age was the peak of the tongue pressure value of Japanese men. The maximum tongue pressure value during the lifetime was found to be 5 to $22 \mathrm{kPa}$ higher in men than for women. However in the case of women the peak value of tongue pressure is keep up to sixties. After that the drop in women's tongue pressure is gentle compared to men. In case of Japanese men, tongue pressure began to decrease after forties, became almost equal tongue pressure to women in seventies, revealed to be below to women after octogenarian.

The Ministry of Health, Labor and Welfare announced that the healthy life expectancy of Japanese in 2016 was 72.14 years for men and 74.79 years for women, with an average life expectancy of 80.98 years for men and 87.14 years for women [20]. Figure 1-3 are combined as one figure, and Figure 4 shows the Japanese healthy life expectancy and average life expectancy is shown in Figure 4. It is one of the characteristics of the Japanese that the healthy life expectancy and the average life expectancy are long. In this Figure 4 shows data of men's tongue pressure data are blue color, data of women are red color, mixed data of men and women are yellow color.

Decrease in tongue pressure value causes dysphagia. Dysphagia of elderly people causes dehydration, malnutrition, suffocation, aspiration pneumonia, and it is one of life threatening factors [21-22]. All elderly people with a tongue pressure of $30 \mathrm{kPa}$ or more ingest a solid meal, and when the tongue pressure is less than 25 to $30 \mathrm{kPa}, 83 \%$ of those who take a solid meal are reduced, and when the tongue pressure is less than $20 \mathrm{kPa}$ There is a report that form-adjusted meals are higher than solid foods [23]. Even in this literature review, the tongue pressure of adults younger than healthy life was maintained at $30 \mathrm{kPa}$ or more. The tongue pressure of adults younger than the average life span was maintained at $25 \mathrm{kPa}$ or more. It is also possible that a drop in tongue pressure may have an effect on the average life expectancy of Japanese health life. The decrease in tongue pressure value in the elderly is the result of natural aging and muscle mass reduction. When thinking about swallowing by the elderly, it is necessary to carefully observe the transition so that the tongue pressure does not fall below $25-30 \mathrm{kPa}$.

\section{Conclusion}

Aim of this literature review was to clarify the features of tongue pressure of healthy Japanese and research problems 
from the viewpoint of gender and age. There were three problems with tongue pressure study.

1) There was no paper showing data from 3 months to the lower grades of elementary school.

2) There are fewer paper than teenagers and nonagenarian.

3) It is necessary to measure the tongue pressure by gender from around the time of the difference in the growth of boys and girls.

The tongue pressure of healthy Japanese had the following characteristics. In the case of women after adulthood, tongue pressure does not become higher than men, but the decrease in tongue pressure value is gradual compared to men with same period. After octogenarian, women showed higher tongue pressure than men. It is also necessary to carefully examine whether tongue pressure is related to healthy life expectancy and life expectancy. Especially in the elderly, attention must be paid to the change of the tongue pressure value so as not to cause trouble in swallowing.

\section{References}

[1] Chen S, Cai Y, Chen F. Lip closing force of ClassIIIpatients with mandibular prognathism: a case control study, Head Face Med, 2014; 10 (33).

[2] Marik PK, Kaplan D. Aspiration pneumonia and dysphagia in the elderly. Chest, 2003; 124: 328-336.

[3] Jung MH, Yang WS, Nahm DS. Effects of upper lip closing force on craniofacial structures, Am J Ortho d, 2003; 123: $58-63$.

[4] Kaede K, Kato T, Yamaguchi M, Nakamura N, Yamada K, Masuda Y. Effects of lip-closing on maximum voluntary lip-closing force during lip pursing in healthy young adults, $\mathrm{J}$ Oral Rehabil, 2016; 43: 169-175.

[5] Nakatsuka K, Adachi T, Kato T, Oishi M, Murakami M, Okada Y, Masuda Y. Reliability of novel multidirectional lip-closing force measurement system, J Oral Rehabil, 2011; 38 18-26.

[6] Shiono Y, Morikawa K, Maki K. Comparative clinical study evaluating lip-closure forces in association with tongue pressure in children, Pediatr Dent J, 2015; 25: 19-25.

[7] Higuchi T, Shiono Y, Maki K et al. Relationship between lip closure force and oral function, Pediatric dentistry J, 2017; 55 (1) 1-10. (In Japanese).

[8] Satomi Y. Maximum tongue pressure measurement by JMS tongue pressure measuring device, Pediatric dentistry J, 2017; 55 (1): 75-75. (In Japanese).

[9] Tada T. Evaluation of oral function by tongue pressure measuring device and examination of tongue training effect, $\mathrm{J}$ Japan Society of Laboratory Automation Chemistry, 2017; 42 (4) 543-543. (In Japanese).
[10] Tamura T, Sato K. New indicators using tongue pressure measuring device in evaluation of articulatory function Examination by young healthy adults -, Niigata Medical Welfare Society J, 2017; 17 (1): 32-32. (In Japanese).

[11] K Tsuga. Functional oral rehabilitation on tongue pressure examination in the elderly people. Ann Jpn Prosthodont Soc, 2016; 8: 52-57.

[12] Satake A, Inui A, Koyama T et al. Oral function test of elderly living in the community - especially with regard to tongue pressure and oral diadogokinesis -, Physical fitness-nutrition-immunology J, 2016; 26 (2): 114-118 (In Japanese).

[13] T Honjyo, K Morikawa, K Saeki, S Nagao, O Hideshima, K Maki. A Study of the Relationship Between Lip Closing Force and Tongue Presser in Children -Comparison of Normal Occlusion Children and Open Bite Children, J Pediatric dentistry. 2015; 53 (1): 60-68.

[14] Fukuchi Y, Mukai S, Yamamoto I et al. Tongue pressure and neonatal prosthesis, J Japanese Academy of Tongue Adhesion, 2014; 20: 11-14 (In Japanese).

[15] Utanohara Y, Hayashi R, Yoshikawa M, et al. Standard values of maximum tongue pressure taken using newly developed disposable tongue pressure measurement device, Dysphagia, 2008; 23: 286-290.

[16] Tsuga K, Maruyama M, Yoshikawa M, Yoshida M, Akagawa Y. Manometric evaluation of oral function with a hand-held balloon probe. J Oral Rehabil, 2011; 38: 680-685.

[17] Yoshida M, Kikutani T, Tsuga K, Utanohara Y, Hayashi R, Akagawa Y. Decreased tongue pressure reflects symptom of dysphagia. Dysphagia, 2006; 21: 61-65.

[18] M Kodama, T Kikutani, M Yoshida, S Inaba. Relationship between Tongue Pressure and Malnutrition in the Institutionalized Elderly, Gerontological dentistry J, 2004; 19 (3) 161-168. (In Japanese).

[19] Ooki S. Statistical genetic analysis of physical developmental longitudinal data at school age, Anthropological Science, 2002; 109 (2): 119-132. (In Japanese).

[20] Ministry of Health Labour and Welfare Japan. http://www.mhlw.go.jp/file/05-Shingikai-10601000-Daijinkan boukouseikagakuka-Kouseikagakuka/0000166296_6.pdf. Retrieved April 18, 2018.

[21] Hudson HM, Daubert CR, Mills RH. The interdependency of protein-energy malnutrition, aging, and dysphagia. Dysphagia 2000; 15:31-38.

[22] Marik PK, Kaplan D. Aspiration pneumonia and dysphagia in the elderly. Chest, 2003; 124: 328-336.

[23] Tanaka Y, Kayashita $J$ et al. On the relation between meal form, tongue pressure, grip strength and walking ability for inpatient and elderly welfare facility residents, Japan Feeding Swallowing Rehabilitation Society J, 2015; 19 (1):52-62. (In Japanese). 